\title{
The Inflaton Field as Self-Interacting Dark Matter in the Braneworld Scenario
}

\author{
James E. Lidsey ${ }^{1}$, Tonatiuh Matos ${ }^{2}$ and L. Arturo Ureña-López ${ }^{2}$ \\ ${ }^{1}$ Astronomy Unit, School of Mathematical Sciences, Queen Mary, University of London, Mile End Road, London E1 4NS, \\ United Kingdom. \\ ${ }^{2}$ Departamento de Física, Centro de Investigación y de Estudios Avanzados del IPN, A.P. 14-740, 07000 México D.F., \\ México.
}

(October 23, 2018)

\begin{abstract}
A unified model is developed within the context of the braneworld paradigm, where a single scalar field can act as both the inflaton field in the very early universe and also as strong, self-interacting dark matter in the post-inflationary universe. Reheating proceeds due to the overproduction and subsequent evaporation of primordial black holes. Observational constraints, most notably from gravitational waves, are satisfied if the probability of PBH formation is sufficiently high.
\end{abstract}

95.35.+d, 98.80.-k

The recent advances in astronomical observations are casting new light on old problems in cosmology. Two of the most fundamental questions of interest today are: (i) the origin of the scalar inflaton field responsible for the accelerated, inflationary expansion of the very early universe and: (ii) the nature of dark matter in the universe. Both questions have important implications for our understanding of large-scale structure formation [1].

New ideas about inflationary cosmology are now emerging with the development of the braneworld scenario, where our observable universe is viewed as a domain wall embedded within a higher-dimensional space [2.3. A striking feature of this scenario is the presence of a quadratic density term in the Friedmann equation [ $⿴ 囗 十$. Under quite general conditions, this term allows steep scalar field potentials to support an inflationary epoch that would otherwise be impossible in standard cosmology 河, 月, 9 .

An attractive feature of steep inflationary models is that the universe can be naturally reheated by the process of gravitational particle production, where particles are produced quantum mechanically at the end of inflation due to the time-variation of the gravitational field [10]. If the inflaton is stable and is able to survive through to the present epoch, it may represent a possible candidate for the cosmological constant or quintessence field [13,6 [9] that has been proposed to account for the high redshift type Ia supernova data [11,12].

On the other hand, Barshay and Kreyerhoff first proposed that a metastable inflaton field could be identified with the present-day cold dark matter (CDM) [13]. By employing renormalization-group techniques to calculate radiative corrections to a scalar field potential involving a quartic self-interaction [14], these authors found that inflation could proceed in the vicinity of the potential's maximum and would end as the field reached a welldefined minimum. Fluctuations about this minimum result in massive inflaton quanta with an energy density that is sufficiently high that they can provide a significant fraction of the CDM at the present epoch.
In this paper, we consider whether the inflaton field can be identified with the cold dark matter in the universe [13], within the specific context of the braneworld scenario and the so-called 'strong, self-interacting scalar field dark matter' (SFDM) hypothesis that has recently been developed by two of the authors 115 21 (see also (22). The key idea of the SFDM scenario is that the dark matter responsible for structure formation in the universe is a real scalar field, $\Phi$, that is minimally coupled to Einstein gravity and has self-interactions parametrized by a potential energy of the form

$$
V(\Phi)=V_{0}\left[\cosh \left(\alpha \sqrt{\kappa_{0}} \Phi\right)-1\right],
$$

where $V_{0}$ and $\alpha$ are the only two free parameters of the model, $\kappa_{0}=8 \pi G$ and we employ natural units such that $\hbar=c=1$. The effective mass of the scalar field is given by $m_{\Phi}^{2}=\kappa_{0} V_{0} \alpha^{2}$. A minimal coupling to gravity avoids the strong restrictions imposed by the equivalence principle on scales of the order of the solar system.

The advantage of the SFDM model is that it is insensitive to initial conditions and the field behaves as CDM once it begins to oscillate around the minimum of its potential. In this case, it can be shown 15, 16] that the SFDM model is able to reproduce all the successes of the standard $\Lambda \mathrm{CDM}$ model above galactic scales. Furthermore, it predicts a sharp cut-off in the mass power spectrum due to its quadratic nature, thus explaining the observed dearth of dwarf galaxies, in contrast with the excess predicted by high resolution N-body simulations with standard CDM 16,23]. The strong self-interaction of the scalar field results in the formation of solitonic objects called 'oscillatons', which have a mass of the order of a galaxy but do not exhibit the cusp density profiles characteristic of standard CDM 19 21]. The best-fit model to the cosmological data can be deduced from the current densities of dark matter and radiation in the universe and from the cut-off in the mass power spectrum that constrains the number of dwarf galaxies in clusters. The favoured values for the two free parameters of the potential (11) are found to be [16]: 


$$
\begin{aligned}
\alpha & \simeq 20.28, \\
V_{0} & \simeq\left(3 \times 10^{-27} M_{4}\right)^{4},
\end{aligned}
$$

where $M_{4} \equiv G^{-1 / 2} \approx 10^{-5} \mathrm{~g}$ is the four-dimensional Planck mass. This implies that the effective mass of the scalar field should be $m_{\Phi} \simeq 9.1 \times 10^{-52} M_{4}=1.1 \times 10^{-23}$ $\mathrm{eV}$.

An important feature of the potential (11) is that it is renormalizable and exactly quantizable, although it is presently unknown whether it originates from a fundamental quantum field theory [24]. Furthermore, the scattering cross section by mass of the scalar particles, $\sigma_{2 \rightarrow 2} / m_{\Phi}$, can be constrained from numerical simulations of self-interacting dark matter that avoid high-density dark matter halos [25]. This effectively constrains the renormalization scale, $\Lambda_{\Phi}$, of the potential to be of the order of the Planck mass, $\Lambda_{\Phi} \simeq 1.93 M_{4}=2.15 \times 10^{19}$ $\mathrm{GeV}$ [18]. Such a value is indicative of a possible fundamental origin for the scalar field, which in turn suggests that the strongly, self-interacting scalar field dark matter may also have been present during the inflationary epoch 18]. However, we do not perform quantum field theoretic or semi-classical calculations in this paper.

In view of the high energy scales associated with the very early universe, it is natural to assume that the scalar field was initially displaced from its global minimum, $\kappa_{0} \alpha^{2} \Phi^{2} \gg 1$. In this limit, the potential is well approximated by an exponential function, but from Eq. (2), the self-coupling is too large to support inflationary expansion in a standard cosmological setting. On the other hand, such a potential can drive inflation successfully within the braneworld scenario [7,9]. This follows because the Friedmann equation is modified due to the motion of our observable universe (the domain wall) through the higher-dimensional 'bulk' spacetime. In particular, in the second Randall-Sundrum scenario [3], the Friedmann equation is given by [4]

$$
H^{2}=\frac{\kappa_{0}}{3} \rho\left(1+\frac{\rho}{2 \lambda_{b}}\right)
$$

when appropriate conditions are satisfied, where $H \equiv$ $\dot{a} / a$ is the Hubble parameter, $a$ is the scale factor of the universe, $\rho$ is the energy-density of the inflaton field (assumed to be confined to the brane) and $\lambda_{b}$ is the tension of the brane. Conventional Einstein gravity is recovered in four dimensions when the energy density is significantly lower than the brane tension, $\rho \ll \lambda_{b}$. However, at high energies, the quadratic correction implies that the expansion rate of the brane is enhanced relative to what it would be in a universe governed by Einstein gravity [5]. Thus, the friction acting on the scalar field is increased and inflation driven by a potential of the form (1) is then possible at sufficiently high energies even though $\alpha^{2} \gg 1$.

Braneworld inflation driven by such a potential has been studied in Refs. [7,9]. Recalling the main results, the COBE normalization of the cosmic microwave background $(\mathrm{CMB})$ power spectrum [26] relates the value of the brane tension to the scalar field self-coupling such that $\lambda_{b}^{1 / 4} \alpha^{3 / 2} \approx 10^{15} \mathrm{GeV}$. For the favoured value of the latter, as implied by Eq. (2), we deduce that

$$
\lambda_{b} \simeq\left(6 \times 10^{-7} M_{4}\right)^{4}=2.88 \times 10^{51} \mathrm{GeV}^{4} .
$$

For these given values of $\left\{\alpha, \lambda_{b}\right\}$, the magnitude of the potential energy at the end of inflation is $V_{\text {end }} \simeq$ $\left(3.2 \times 10^{-6} M_{4}\right)^{4}=2.33 \times 10^{54} \mathrm{GeV}^{4}$ and this implies that $\Phi_{\text {end }} \approx 2 M_{4}$, thus justifying the exponential approximation to the potential (11) during the inflationary era. In comparison with the canonical $V=\lambda \Phi^{4}$ potential, where COBE normalization implies the dimensionless parameter $\lambda \approx 10^{-13}$ should be very small, our model requires the small dimensionful number for $V_{0}$ given in Eq. (3), although this constraint does not arise from the CMB. Furthermore, the magnitude of the potential $N e^{-}$ foldings before the end of inflation is $V_{N} \approx(N+1) V_{\text {end }}$, implying that the initial value of the scalar field is closer to $\Phi_{\text {end }}$ than in the quartic model.

Given the COBE normalization [26], the spectral index of the scalar fluctuation spectrum is determined to be [7]

$$
n=1-\frac{4}{N+1}=0.94,
$$

where $N \approx 70$ is the number of $e$-foldings that elapse between the epoch that a given, observable mode crosses the Hubble radius during inflation and the end of the inflationary epoch. Remarkably, the tilt of the scalar perturbation spectrum in this scenario is uniquely determined by the number of $e$-foldings and is independent of the parameters in the potential (11). A spectrum with a tilt of this magnitude away from scale-invariance is presently favoured by analyses of the CMB power spectrum [27]. Furthermore, the amplitude of the primordial gravitational wave spectrum, $A_{T}$, relative to that of the density perturbations, $A_{S}$, can be estimated as [7]

$$
r=4 \pi \frac{A_{T}^{2}}{A_{S}^{2}} \simeq 0.4
$$

implying after COBE normalization that $A_{T}^{2} \approx 1.7 \times$ $10^{-10}$. This ratio is also independent of the model's parameters and is within the projected sensitivity of the Planck satellite. It provides a potentially powerful test of the model.

Inflation ends when the quadratic corrections to the Friedmann equation (4) become negligible. Due to the steep nature of its potential, the inflaton then behaves as a massless field, where its energy density redshifts as $\rho_{\Phi} \propto a^{-6}$. This is important, because after the tensor modes have re-entered the Hubble radius, the evolution of the spectral gravity wave energy density, $\tilde{\rho}_{g}$, is sensitive to the effective equation of state in the postinflationary universe [9,28]. It is enhanced (reduced) on

${ }^{*}$ The equation of state is assumed to be of the form $p=\omega \rho$, 
shorter scales if $\omega>1 / 3(\omega<1 / 3)$. In general, the bound on the gravitational waves imposed by successful nucleosynthesis, $\rho_{g} \leq 0.2 \rho_{\text {rad }}$, must not be violated and this results in an upper limit on the duration of the kinetic energy dominated phase [9].

Sahni, Sami and Souradeep [9] assume that the evolution of the short wavelenth gravitational waves is similar to that of conventional cosmology. They then conclude that the gravitational wave energy density begins to dominate the scalar field when the universe has expanded by a factor of $A_{T}^{-1} \approx 10^{5}$ and, consequently, the universe must become radiation dominated before the temperature has fallen by this factor. Unfortunately, for the model under consideration, the thermalized temperature of the radiation produced from gravitational particle production at the end of inflation is given by $T_{\text {end }} \simeq 2 \times 10^{9} \mathrm{GeV}$, whereas the temperature at the epoch when this radiation dominates the scalar field is $T_{\text {eq }} \simeq(1-2) \mathrm{GeV}$ [9], corresponding to a redshift of $10^{9}$.

An alternative mechanism for reheating the universe is therefore required that reduces the duration of the kinetic energy dominated phase. One possibility is through the overproduction of primordial black holes (PBHs) that subsequently decay into relativistic particles via Hawking evaporation 29 31]. In the above inflationary model, a fraction, $\beta_{0}$, of the energy density of the universe collapses into PBHs due to the density fluctuations that re-enter the Hubble radius immediately after the universe has ceased to accelerate. The $\mathrm{PBHs}$ form with a mass of the order of the horizon mass at this time [32] and this is given by $M_{\mathrm{pbh}} \approx M_{4}^{2} H_{\text {end }}^{-1}$, where the Hubble radius at the end of inflation, $H_{\text {end }}^{-1}$, is estimated from the Friedmann equation (位) under the assumption that $\rho_{\text {end }} \approx V_{\text {end }} \approx 2 \alpha^{2} \lambda_{b}$. Eq. (5) then implies that $M_{\mathrm{pbh}} \approx 10^{9} M_{4}$ and the lifetime of $\mathrm{PBHs}$ with this mass is $t_{\text {evap }} \approx\left(M_{\mathrm{pbh}} / M_{4}\right)^{3} t_{P} \approx 10^{-16} \mathrm{~s}$, where $t_{P}$ is the Planck time. This is sufficiently short that constraints on PBH evaporations from primordial nucleosynthesis are satisfied [30.33].

Once formed, the PBHs behave as a pressureless fluid and their energy density redshifts as $\rho_{\mathrm{pbh}} \propto a^{-3}$. Thus, they are able to dominate the (massless) scalar field before they evaporate if

$$
\frac{\beta_{0}}{1-\beta_{0}}>\left(\frac{M_{\mathrm{pbh}}}{M_{4}}\right)^{-2} \approx 10^{-18}
$$

and this change in the effective equation of state occurs after the universe has expanded by a factor

$$
\frac{\beta_{0}}{1-\beta_{0}} \approx\left(\frac{a_{\mathrm{end}}}{a_{\mathrm{dom}}}\right)^{3}
$$

where $\omega$ is a constant barotropic index. For a massless scalar field, $\omega=1$. where $a_{\mathrm{dom}}$ denotes the scale factor at the onset of PBH domination. It follows, therefore, that the PBHs dominate the scalar field before the backreaction of the gravitational waves becomes significant if the initial mass fraction satisfies $\beta_{0}>10^{-15}$. This is consistent with Eq. (8) and the constraint arising from the integrated gravitational wave energy density is therefore alleviated since the PBH equation of state, $\omega=0$, is 'softer' than that of radiation.

A stronger, and more reliable, constraint on the initial $\mathrm{PBH}$ fraction can be imposed by requiring that the PBHs dominate the universe before the scalar field has reached the minimum of its potential and begun to oscillate. During the kinetic dominated regime, the scalar field varies as

$$
\Phi=\Phi_{\mathrm{end}}-\sqrt{\frac{3}{4 \pi}} M_{4} \ln \left(\frac{a}{a_{\mathrm{end}}}\right)
$$

and from the estimate of $\Phi_{\text {end }}$ given above, $\Phi_{\text {end }} \approx 2 M_{4}$, the field reaches the minimum of its potential after the universe has redshifted by a factor $a / a_{\text {end }} \approx 60$. Thus, from Eq. (9), the PBHs dominate the universe before this point is reached if $\beta_{0}>5 \times 10^{-6}$.

If the PBHs come to dominate sufficiently early, the displacement of the scalar field away from its minimum is such that the potential is still well approximated by an exponential form at this time. This is the case for $\Phi>$ $0.01 M_{4}$. Moreover, the standard Friedmann equation is valid for $\Phi<1.8 M_{4}$, where the quadratic corrections in Eq. (4) become negligible. Since its potential is steep, the field subsequently tracks the $\mathrm{PBH}$ (fluid) component in this regime as in the standard cosmology [34], where its potential and kinetic energies scale at the same rate as that of the PBH energy density. More specifically, $\Omega_{\Phi}=3(1+\omega) / \alpha^{2}$ and $\dot{\Phi}^{2} / V=2(1+\omega) /(1-\omega)$ and this implies that the variation of the scalar field with respect to the scale factor during tracking is given by [34]

$$
\Phi=\Phi_{\mathrm{t} 0}-\frac{3(1+\omega)}{\sqrt{\kappa_{0}} \alpha} \ln \left(\frac{a}{a_{\mathrm{t} 0}}\right)
$$

where a subscript ' $\mathrm{t} 0$ ' denotes the onset of the tracking behaviour. This is important because the large value of the field's self-coupling, $\alpha \approx 20$, implies that the universe expands by many orders of magnitude before the field reaches its global minimum. In particular, if the PBHs dominate the cosmic dynamics for the majority of their lifetime, the universe can expand by up to a factor of $10^{12}$ before the PBHs evaporate. The rolling of the scalar field down its potential during this epoch is only $\Delta \Phi \approx-0.84$. The subsequent transition to a radiation dominated universe has a negligible effect on the tracking behaviour of the field, and for a wide range of initial conditions, the field does not reach its minimum until well after the primordial nucleosynthesis era has passed. In this case, the nucleosynthesis bounds are not violated since $\alpha>5$ [35]. In principle, therefore, our model does 
not exhibit the problem of overshooting nor undershooting for the initial conditions [35]. The post-inflationary universe after the nucleosynthesis era would then correspond to the universe considered in Refs. [15 18], where it was shown that the scalar field can subsequently act as dark matter in the universe.

To summarize, we have found that a scalar field with a potential of the form (11) can drive an epoch of inflationary expansion in the braneworld scenario and may also act as a candidate for the dark matter at the present epoch. The reheating of the universe proceeds via $\mathrm{PBH}$ domination and evaporation. The model is able to explain a variety of astrophysical observations over a wide range of scales and, indeed, contains only four, free parameters: the effective mass of the scalar field, $m_{\Phi}$, its self-coupling, $\alpha$, the tension of the brane, $\lambda_{b}$, and the initial mass fraction of PBHs, $\beta_{0}$. It is the insensitivity of Eqs. (6) and (7) to the potential's parameters that allows us to simultaneously satisfy the constraints arising from the mass power spectrum without the need for finetuning. Once fixed by observational constraints, the parameters of the potential can remain unaltered throughout the history of the universe.

In this paper, we have considered the potential (1) from a phenomenological perspective with the primary aim of determining the region of parameter space consistent with astrophysical observation. The parameters cover a wide range of scales and one question that immediately arises is whether the form of the potential (11) and, in particular, the best-fit values for its parameters, can be more fully understood from a field-theoretic perspective. This is beyond the scope of the present paper. Nevertheless, we have developed a model based on a single scalar field that covers the history of the universe from the inflationary era through to the present epoch and it is therefore to be expected that the parameters should cover a similar range of energy scales. The success of the model in simultaneously satisfying a number of fundamental observational constraints provides strong motivation for considering its field-theoretic origin in more detail.

We have viewed the initial mass fraction of $\mathrm{PBHs}$ as a free parameter in the above analysis, but its magnitude is determined by the density perturbations at the end of inflation. A more detailed calculation of the fluctuation spectra is therefore required if further insight is to be gained, but this involves an extension of the slow-roll analyses employed thus far [5] and is beyond the scope of the present paper. Since the post-inflationary universe is initially dominated by a scalar field, the question of $\mathrm{PBH}$ formation in this scenario is closely related to the problem of scalar field collapse and this topic has attracted considerable attention in recent years. (For a recent review, see, e.g., Ref. 36]). Moreover, in estimating the limits on the probability of $\mathrm{PBH}$ formation, we have assumed that standard four-dimensional results derived within the context of Einstein gravity are valid. This is reasonable since the $\mathrm{PBH}$ form once inflation has ended and this occurs when the brane corrections to the Friedmann equation (4) have become negligible.

A potential problem with reheating a braneworld inflationary universe via $\mathrm{PBH}$ evaporations is that the decay products may radiate primarily off the brane and into the higher-dimensional bulk, thereby rendering the brane effectively cold and empty. This remains an open question in the second Randall-Sundrum scenario, but it has been shown in a related model that most of the Hawking radiation consists of standard model particles that are indeed confined to the brane 37].

Finally, there is the question of whether the $\mathrm{PBH}$ leave behind stable, Planck-sized relics at the endpoint of their evaporation 30.38. Although such a possibility is now considered unlikely, it is worth remarking that when the $\mathrm{PBH}$ dominate the universe before they evaporate, their relics can not overclose the universe if $M_{\mathrm{pbh}}<10^{6} \mathrm{~g} 30$ and this bound is satisfied for the above scenario.

In conclusion, we have proposed a simple, unified model of the inflaton and dark matter particles, where the same scalar field provides the origin for the primordial spectrum of density perturbations produced quantum mechanically during inflation and also plays a central role in forming the cosmological structures that we observe today.

\section{ACKNOWLEDGMENTS}

JEL is supported by the Royal Society. LAUL wants to thank the Theoretisch-Physikalisches Institut (Jena) for its kind hospitality. TM and LAUL are partly supported by CONACyT México, under grants 119259 (L.A.U.) and 34407-E. JEL's visit to CINVESTAV was supported by CONACyT grant 38495-E. We thank C. TerreroEscalante for a helpful discussion.

[1] A. R. Liddle and D. H. Lyth, Cosmological Inflation and Large Scale Structure (Cambridge University Press, Cambridge, 2000).

[2] K. Akama, hep-th/0001113, V. A. Rubakov and M. E. Shaposhnikov, Phys. Lett. 159B, 22 (1985); N. ArkaniHamed, S. Dimopoulos, and G. Dvali, Phys. Lett. B 429, 263 (1998); M. Gobverashvili, Europhys. Lett. 49, 396 (2000); L. Randall and R. Sundrum, Phys. Rev. Lett. 83, 3370 (1999).

[3] L. Randall and R. Sundrum, Phys. Rev. Lett. 83, 4690 (1999).

[4] P. Kraus, J. High En. Phys. 9912, 011 (1999); D. Ida, J. High En. Phys. 0009, 014 (2000); C. Barcelo and M. Visser, Phys. Lett. B482, 183 (2000); P. Binétruy, C. Deffayet, U. Ellwanger, and D. Langlois, Phys. Lett. B477, 285 (2000); E. E. Flanagan, S. -H. Tye, and I. 
Wasserman, Phys. Rev. D 62, 044039 (2000); C. Csaki, M. Graesser, C. Kolda, and J. Terning, Phys. Lett. B462, 34 (1999); J. M. Cline, C. Grojean, and G. Servant, Phys. Rev. Lett. 83, 4245 (1999).

[5] R. Maartens, D. Wands, B. A. Basset, and I. P. C. Heard, Phys. Rev. D 62, 041301 (2000).

[6] P. J. E. Peebles and A. Vilenkin, Phys. Rev. D 59, 063505 (1999); R. A. Frewin and J. E. Lidsey, Int. J. Mod. Phys. D 2, 323 (1993); M. Giovannini, Phys. Rev. D 60, 123511 (1999); M. Peloso and F. Rosati, J. High En. Phys. 9912, 026 (1999); F. Rosati, hep-ph/0002090; K. Dimopoulos, astro-ph/0012298; A. S. Majumdar, Phys. Rev. D 64, 083503 (2001); A. B. Kaganovich, Phys. Rev. D 63. 025022 (2001); K. Dimopoulos and J. W. F. Valle, astroph/0111417; K. Dimopoulos, astro-ph/0111500.

[7] E. J. Copeland, A. R. Liddle, and J. E. Lidsey, Phys. Rev. D 64, 023509 (2001).

[8] G. Huey and J. Lidsey, Phys. Lett. B514, 217 (2001).

[9] V. Sahni, M. Sami, and T. Souradeep, gr-qc/0105121.

[10] L. H. Ford, Phys. Rev. D 35, 2955 (1987); L. P. Grishchuk and Y. V. Sidorov, Phys. Rev. D 42, 341 (1990); B. Spokoiny, Phys. Lett. B 315, 40 (1993).

[11] R. R. Caldwell, R. Dave, and P. J. Steinhardt, Phys. Rev. Lett. 80, 1582 (1998); L. Wang, R. R. Caldwell, J. P. Ostriker, and P. J. Steinhardt, Astrophys. J. 503, 17 (2000).

[12] B. P. Schmidt, et al., Astrophys. J. 507, 46 (1998); A. G. Riess, et al., Astron. J. 116, 1009 (1998); S. Perlmutter et al., Astrophys. J. 517, 565 (1999).

[13] S. Barshay and G. Kreyerhoff, Eur. Phys. J. C 5, 369 (1998).

[14] S. Barshay and G. Kreyerhoff, Z. Phys. C 75, 167 (1997); Errat. Z. Phys. C 76, 577 (1997).

[15] T. Matos and L. A. Ureña-López, Class. Quantum Grav. 17, L75 (2000).

[16] T. Matos and L. A. Ureña-López, Phys. Rev. D 63, 063506 (2001).

[17] T. Matos and F. S. Guzmán, Class. Quantum Grav. 18, 5055 (2001).

[18] T. Matos and L. A. Ureña-López, astro-ph/0010226.

[19] L. A. Ureña-López, gr-qc/0104093; L. A. Ureña-López, T. Matos, and R. Becerril, in preparation.

[20] M. Alcubierre, T. Matos, and D. Núñez, in preparation.

[21] M. Alcubierre, F. S. Guzmán, T. Matos, D. Núñez, L. A. Ureña-López, and P. Wiederhold, gr-qc/0110102.

[22] V. Sahni and L. Wang, Phys. Rev. D 62, 103517 (2000).

[23] M. Kamionkowski and A. R. Liddle, Phys. Rev. Lett. 84, 4525 (2000); J. R. Primack, astro-ph/0112255; P. Salucci and A. Borriello, astro-ph/0011079 B. Moore, astro-ph/0103100.

[24] K. Halpern and K. Huang, Phys. Rev. Lett. 74, 3526 (1995); K. Halpern and K. Huang, Phys. Rev. D 53, 3252 (1996). Vincenzo Branchina, Phys. Rev. D 64043513 (2001). C. Bagnuls and C. Bervillier, Phys. Rept. 384, 91 (2001).

[25] D. N. Spergel and P. J. Steinhardt, Phys. Rev. Lett. 84, 3760 (2000); B. D. Wandelt, R. Dave, G. R. Farrar, P. C. McGuire, D. N. Spergel, and P. J. Steinhardt, astro-ph/0006344; C. Firmani, E. D'Onghia, V. AvilaReese, G. Chincarini, and X. Hernández, MNRAS 315,
L29 (2000); C. Firmani, E. D’Onghia, G. Chincarini, X. Hernández, and V. Avila-Reese, MNRAS 321, 713 (2001); M. Kaplinghat, L. Knox, and M. S. Turner, Phys. Rev. Lett. 85, 3335 (2000).

[26] E. F. Bunn, A. R. Liddle, and M. White, Phys. Rev. D 54, 5917R (1996); E. F. Bunn and M. White, Astrophys. J. 480, 6 (1997).

[27] X. Wang, M. Tegmark, and M. Zaldarriaga, astro$\mathrm{ph} / 0105091$.

[28] B. Allen, Phys. Rev. D 37, 2078 (1988); V. Sahni, Phys. Rev. D 42, 453 (1990).

[29] S. W. Hawking, Nat. 248, 30 (1974); Commun. Math. Phys. 43, 199 (1975).

[30] B. J. Carr and J. E. Lidsey, Phys. Rev. D 48, 543 (1993); B. J. Carr, J. H. Gilbert, and J. E. Lidsey, Phys. Rev D 50, 4853 (1994).

[31] M. Yu. Khlopov, B. A. Malomed, and Ya. B. Zel'dovich, Mon. Not. R. Astron. Soc. 215, 575 (1985); P. D. Nasel'skii and A. G. Polnarev, Sov. Astron. 29, 487 (1985); J. D. Barrow, E. J. Copeland, E. W. Kolb, and A. R. Liddle, Phys. Rev. D 43, 984 (1991); J. GarcìBellido, A. D. Linde, and D. Wands, Phys. Rev. D 54, 6040 (1996); L. Randall, M. Soljacic, and A. H. Guth, Nucl. Phys. B472, 377 (1996); A. M. Green and K. A. Malik, Phys. Rev. D 64, 021301 (2001).

[32] B. J. Carr, Astrophys. J. 205, 1 (1975).

[33] A. M. Green and A. R. Liddle, Phys. Rev. D 56, 6166 (1997).

[34] C. Wetterich, Nucl. Phys. B302, 668 (1988); B. Ratra and P. J. E. Peebles, Phys. Rev. D 37, 3406 (1988); E. J. Copeland, A. R. Liddle, and D. Wands, Phys. Rev. D 57, 4686 (1998).

[35] P. G. Ferreira and M. Joyce, Phys. Rev. D 58, 023503 (1998).

[36] A. Wang, Braz. J. Phys. 31, 188 (2001) gr-qc/0104073.

[37] R. Emparan, G. T. Horowitz, and R. C. Myers, Phys. Rev. Lett. 85, 499 (2000).

[38] J. D. Barrow, E. J. Copeland, and A. R. Liddle, Phys. Rev. D 46, 645 (1992). 